\title{
The Structure of the Forward Peak in Elastic and Inelastic Diffraction of Hadrons
}

\author{
Andrzej R. Małecki* \\ KEN Pedagogical Academy, Kraków, Poland \\ e-mail: amalecki@ap.krakow.pl
}

Elastic scattering and inclusive inelastic diffraction of high energy hadrons at small momentum transfers are considered. A general expression for the elastic scattering amplitude in terms of even moments of the probability distribution is presented. We also show that the shape of the forward inclusive inelastic diffraction is determined by elastic scattering in the transition region between the forward peak and diffraction minimum.

International Workshop on Diffraction in High-Energy Physics -DIFFRACTION 2006 -

September 5-10 2006

Adamantas, Milos island, Greece

* Speaker. 


\section{Elastic scattering amplitude and the moments of hadronic density}

The familiar form of the hadron elastic scattering amplitude $T(s, t)$ at high energies is given as the 2-dimensional probability Fourier transform of the 1-dimensional probability $1-S(s, b)$ :

$$
T(s, t)=\frac{i}{2 \pi} \int d^{2} b e^{i \vec{q} \cdot \vec{b}}[1-S(s, b)]
$$

where the squared momentum transfer $t=\vec{q}^{2}$ and the c.m. energy equals $\sqrt{s}$.

Even if $T(s, t)$ is not the generator of the moments of the probability distribution $1-S$, nevertheless the even moments of this distribution can be obtained from $T(s, t)$. For this scope let us consider the Laplace operator:

$$
\triangle=\nabla^{2}=\sum_{j=1}^{2} \frac{\partial^{2}}{\partial q^{j} \partial q^{j}}=-4\left(\frac{d}{d t}+t \frac{d^{2}}{d t^{2}}\right)
$$

and apply it $n$ times to both sides of (1.1).

We have at $t=0$ on the left and right hand side, respectively:

$$
\triangle^{n} T(s, t=0)=2^{2 n} n ! \frac{d^{n} T(s, t=0)}{d t^{n}}=\frac{i}{4 \pi}(-1)^{n} \frac{M_{2 n}}{\mu^{2 n+2}}
$$

where the dimensionless, energy dependent, even moments of the probability distribution $1-S$ are defined as:

$$
M_{2 n}(s)=4 \pi \int d(\mu b)(\mu b)^{2 n+1}[1-S(\kappa(s), \mu b)]
$$

with the parameters $\kappa(s)$ and $\mu$ describing the dependence of the profile $1-S$ on energy and the impact parameter, respectively.

The even moments $M_{2 n}(s)$ are related to $T=1-S$ by decreasing functions of $\kappa(s)$. Consequently, the moments $M_{2 n}(s)$ are increasing functions of $s$, since $\kappa(s)$ must increase with $s$. This behaviour is different from that of the moments of a classical geometrical model of a black disk which are constants. It is instructive to remind this well known result since the leading term in the asymptotic expansion of the famous Chou-Yang S-matrix in b-space is just a black disc S-matrix.

The Taylor expansion of the amplitude $T(s, t)$ about $t=0$ follows from (1.3). We have

$$
T(s, t)=\frac{i}{4 \pi \mu^{2}} \sum_{n=0}^{\infty} \frac{M_{2 n}(s)}{(n !)^{2}}\left(-\frac{t}{4 \mu^{2}}\right)^{n}
$$

where from, in particular, the total cross-section is $\sigma_{t o t}(s)=M_{0}(s) / \mu^{2}$ and the forward slope parameter equals $\beta(s)=M_{2}(s) / 2 M_{0} \mu^{2}$.

It is useful to consider the generator of the moments $M_{2 n}(s)$ defined as:

$$
G(s, t)=\frac{i}{4 \pi \mu^{2}} 2 \pi \int_{0}^{\infty} d z \exp \left(\frac{i t z}{4 \mu^{2}}\right)\left[1-S\left(\kappa(s), z=(\mu b)^{2}\right)\right]
$$

whose Taylor series expansion about $t=0$ is

$$
G(s, t)=\frac{i}{4 \pi \mu^{2}} \sum_{n=0}^{\infty} \frac{M_{2 n}(s)}{n !}\left(\frac{i t}{4 \mu^{2}}\right)^{n}
$$


which means that the generator of the moments is the Borel transform of $T(s, t)$. Therefore the convergence of the series implies that the amplitude $T(s, t)$ is an entire function of $t$. It means that $T(s, t)$, being an entire function of $t$, admits a canonical factorization in terms of its zeroes.

When the scattering amplitude $T(s, t)$ has no zeroes it may be expressed in a useful form:

$$
T(s, t)=\frac{i}{4 \pi \mu^{2}} M_{0}(s) \exp \left[\sum_{n=1}^{\infty} \frac{C_{n}(s)}{n !}\left(-\frac{t}{4 \mu^{2}}\right)^{n}\right]
$$

where the coefficients $C_{n}(s)$ are determined in terms of the reduced moments:

$$
N_{n}(s)=\frac{M_{2 n}(s)}{n ! M_{0}(s)}
$$

by comparing the two last expressions for the amplitude $T(s, t)$. For the first few coefficients one finds: $C_{1}=N_{1}, C_{2}=N_{2}-N_{1}^{2}, C_{3}=N_{3}-3 N_{1} N_{2}+2 N_{1}^{3}$.

Let us notice that the approximate form of the amplitude $T(s, t)$ given in (1.8)

$$
T(s, t)=\frac{i}{4 \pi \mu^{2}} M_{0}(s) \exp \left[-C_{1}(s) \frac{t}{4 \mu^{2}}\right]
$$

is well known in relation to the slope parameter $\beta(s)$. In particular, the increase of $\beta(s)$ with energy $\sqrt{s}$ gives rise to the phenomenon of shrinking of the diffraction peak. Recently, A. Martin and his collaborators [1] forwarded arguments that this simple approximation should not be used at the LHC high energies.

\section{Inclusive inelastic cross-section of hadron diffraction}

In the formalism of "diffractive limit" the inelastic diffraction is built as an infinite sum of the infinitesimal contributions from the subspace of diffractive states. The expressions of the form $N \Delta t$, where $\Delta t$ represents diversity of the diagonal matrix elements of the scattering operator $t_{j}$ over the subspace of diffractive states [D], are to be considered in the double diffractive limit [2]: $N \rightarrow \infty, \Delta t \rightarrow 0$ such that $N \Delta t$ is finite.

The inclusive inelastic cross-section arises by making use of completness of diffractive states in their equivalence subspace:

$$
\sum_{|f\rangle \neq|i\rangle}\left|T_{f i}\right|^{2}=N^{2} \sum_{|f\rangle \in[D]}\left|\Lambda_{i f}\right|^{2}\left|t_{a v}^{(i)}-t_{f}\right|^{2}+\left(1-g_{i}\right) g_{i} N^{2}\left|t_{a v}^{(i)}-t_{i}\right|^{2}
$$

Thus the inclusive cross-section of inelastic diffraction is built of the two contributions: an incoherent one which is proportional to a dispersion of the $T_{0}$-diagonal matrix elements and another one which equals (up to a constant) the diffractive contribution to elastic scattering (coherent term):

$$
\frac{1}{2 \pi} \frac{d \sigma_{d i f}(t)}{d|t|}=\sum_{|f\rangle \neq|i\rangle}\left|T_{f i}(q)\right|^{2} \equiv \frac{d \sigma_{\text {incoh }}(q)}{d^{2} q}+\frac{d \sigma_{c o h}(q)}{d^{2} q} .
$$

The coherent cross-section $\sigma_{c o h}$ is proportional to the square of diffractive term in the elastic scattering amplitude:

$$
\frac{d \sigma_{c o h}(q)}{d^{2} q}=\left(\frac{1}{g_{i}}-1\right)\left|T_{i i}(q)-t_{i}(q)\right|^{2}
$$




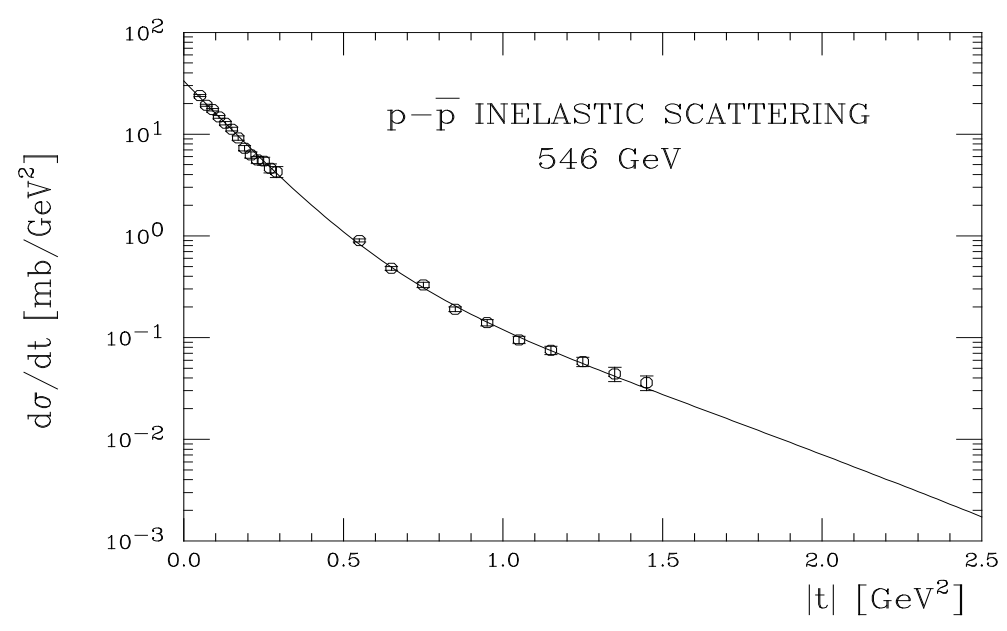

Figure 1: Proton - antiproton inclusive inelastic diffraction at c.m. energy $\sqrt{s}=546 \mathrm{GeV}$ [3].

The name of incoherent contribution is justified by its proportionality, in a leading order, to the mean value $\langle n\rangle$. It appears in the form of the double Fourier-Bessel transform:

$$
\frac{d \sigma_{\text {incoh }}(q)}{d^{2} q}=\frac{1}{(2 \pi)^{2}} \int d^{2} b_{1} d^{2} b_{2} e^{i \vec{q} \cdot\left(\vec{b}_{1}-\vec{b}_{2}\right)}\left[1-\Gamma_{0}\left(b_{1}\right)\right]\left[1-\Gamma_{0}\left(b_{2}\right)\right] I\left(\vec{b}_{1}, \vec{b}_{2}\right)
$$

where the function

$$
I\left(\vec{b}_{1}, \vec{b}_{2}\right)=N^{2} g_{i}\langle n\rangle U\left(\vec{b}_{1}, \vec{b}_{2}\right)
$$

depends on the correlation function of diffractons

$$
U\left(\vec{b}_{1}, \vec{b}_{2}\right) \equiv \int d^{2} s|\psi(s)|^{2} \gamma\left(\vec{b}_{1}-\vec{s}\right) \gamma\left(\vec{b}_{2}-\vec{s}\right) .
$$

Since the major contribution to the integrand function comes from small values of $\vec{b}^{\prime} \equiv \vec{b}_{1}-\vec{b}_{2}$, one may approximate: $\Gamma_{0}\left(b_{1}\right)=\Gamma_{0}\left(b_{2}\right)=\Gamma_{0}(b)$ and $S\left(b_{1}\right)=S\left(b_{2}\right)=S(b)$ where $\vec{b} \equiv \frac{1}{2}\left(\vec{b}_{1}+\vec{b}_{2}\right)$. This allows to convert the integral (2.4) into a sum of products of single integrals.

From the inspection of the above formulae it results that for the description of inclusive inelastic diffraction only 3 parameters $g_{i},\langle n\rangle$ and the diffracton radius $R_{\varepsilon}$ are required since the remaining parameters are to be determined from elastic scattering.

The fit to the experimental data on proton-antiproton inelastic diffraction at c.m.energy $\sqrt{s}=$ $546 \mathrm{GeV}[3]$ (23 points) presented in Fig. 1 is excellent in the whole range of momentum transfer.

The measurements of the inclusive inelastic cross-section at the ISR and SPS colliders [5, 3] are perhaps not sufficiently appreciated. The angular distribution of inelastic diffraction is, in a wide range of energy, consistently characterised by two different slopes at small and large momentum transfers. The experimental results could therefore be well reproduced simply with a sum of two Gaussians described by 4 parameters: two slopes and two other parameters which fix the forward magnitude of each Gaussian. However, in our phenomenology we need only 3 parameters since the slope at small momentum transfers is already determined by the diffractive term in elastic scattering. The strength of this term in inelastic diffraction is set-up by the coupling constant $g_{i}$. So far, this constant was hidden in the definition of the cross-section $\sigma_{n}$ and in inelastic diffraction it appears as a new parameter at disposal. 


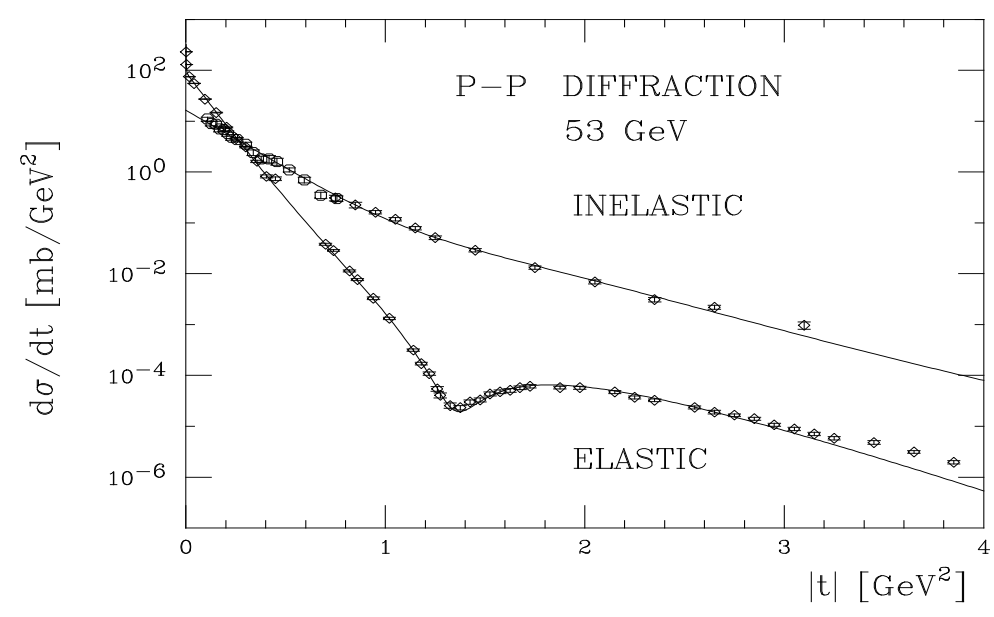

Figure 2: Proton - proton elastic and inclusive inelastic diffraction at c.m. energy $\sqrt{s}=53 \mathrm{GeV}[4,5]$.

\section{Diffractive limit versus "diffractive" eigenstates}

The numerical results presented in Section 2 will be discussed again with emphasis on the comparison of our approach based on the diffractive limit $N \rightarrow \infty$ with the classical description of Good and Walker [6] based on the presumed diagonalization of the scattering operator $T$ in physical states. On the concept of "diffractive eigenstates" are grounded all geometrical models of diffraction, with all their merits and limitations. Indeed, since $T$ is normal it has eigenstates, but the fundamental point in the description of diffraction is the reducibility of the Hilbert space into two orthogonal subspaces of diffractive and non-diffractive states.

In order to understand better the relationship of the two approaches we rewrite their basic formulae using a unified notation. Thus for the elastic scattering amplitudes one has:

$$
\begin{gathered}
T_{i i}=t_{i}+N \sum_{|j\rangle \in[D]}\left|\Lambda_{i j}\right|^{2}\left(t_{j}-t_{i}\right), \\
T_{i i}^{(G W)}=t_{i}+\sum_{|j\rangle \in[D]}\left|\Lambda_{i j}\right|^{2}\left(t_{j}-t_{i}\right) ;
\end{gathered}
$$

while the inclusive inelastic cross-sections read :

$$
\begin{gathered}
\sum_{|f\rangle \neq|i\rangle}\left|T_{f i}\right|^{2}=N^{2} \sum_{|j\rangle \in[D]}\left|\Lambda_{i j}\right|^{2}\left|t_{j}-t_{i}\right|^{2}-\left|T_{i i}-t_{i}\right|^{2}, \\
\sum_{|f\rangle \neq|i\rangle}\left|T_{f i}^{(G W)}\right|^{2}=\sum_{|j\rangle \in[D]}\left|\Lambda_{i j}\right|^{2}\left|t_{j}-t_{i}\right|^{2}-\left|T_{i i}^{(G W)}-t_{i}\right|^{2},
\end{gathered}
$$

where the weigth $\left|\Lambda_{i j}\right|^{2}$ is normalized to $g_{i}$. The two sets of formulae are almost identical except for the infinite factor $N$ which in the Good-Walker approach is absent while the value of $g_{i}$ is fixed to 1 . But these differences turn out to be essential.

It should be stressed that the coherent contribution (2.3) to the inclusive cross-section is a novelty of our approach. In the standard version of the Good-Walker description (with $g_{i} \equiv 1$ ) the coherent contribution to inelastic diffraction does not appear at all. Thus in [7] the forward 


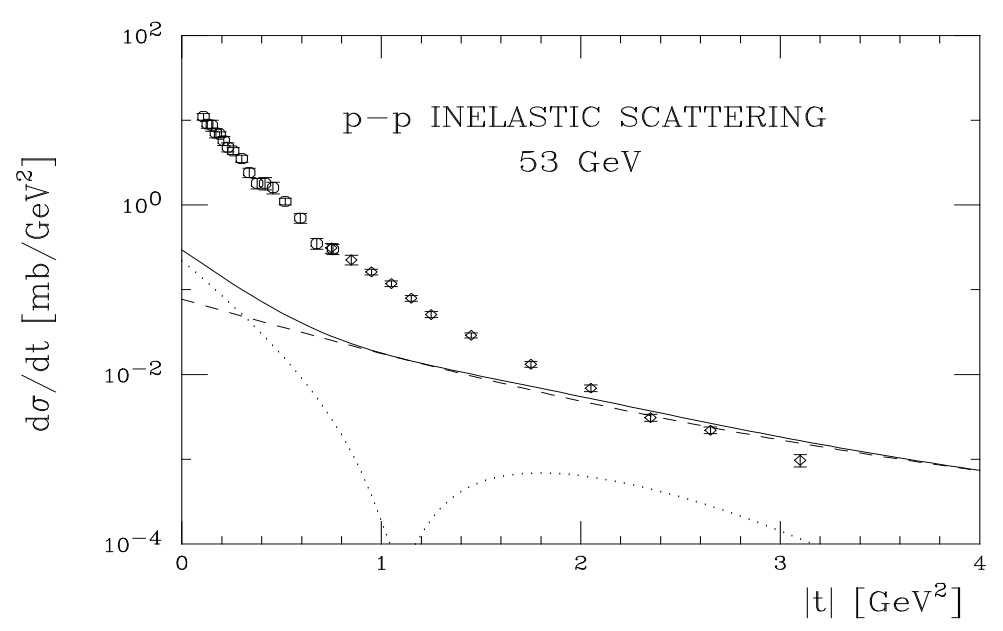

Figure 3: Proton - proton inclusive inelastic diffraction at c.m. energy $\sqrt{s}=53 \mathrm{GeV}$. The experimental data [3] are compared with the Good-Walker approach (solid curve). The coherent (dotted curve) and incoherent (dashed curve) contributions to the cross-section are shown separately.

slope of inelastic diffraction was explained in terms of incoherent scattering while our analysis shows that this scattering dominates in the region of large momentum transfers. We claim that the shape of inelastic diffraction at small momentum transfers is determined by elastic scattering in the transition region between the forward peak and the diffraction minimum. This is successfully verified, as Figs. 1 and 2 show, in experiment [5, 3], being a crucial evidence in favor of our formalism.

On the contrary, the Good - Walker approach, even allowing for $g_{i} \neq 1$, is not able to accomodate this effect. This is so because the coherent contribution to inelastic diffraction is there completely fixed (including the value of $g_{i}$ ) by elastic scattering. The thus determined coherent contribution, being proportional to $\left(1 / g_{i}-1\right) g_{i}^{2}$, turns out, both for small and large values of $g_{i}$, to be too small and does not reproduce the inelastic cross-section at low momentum transfers as illustrated in Fig. 3.

\section{References}

[1] C. Bourrely, N. N. Khuri, A. Martin, J. Soffer and T. T. Wu, preprint of the talk at the 2005 Blois conference.

[2] A. R. Małecki, Nucl. Phys. (Proc. Suppl.) B 146 (2005) 197.

[3] D. Bernard et al., Phys. Lett. B 186 (1987) 227.

[4] K. R. Schubert, Tables of Nucleon - Nucleon Scattering, Landolt-Bornstein, New Series, Vol. 1/9a, Springer, Berlin, 1979.

[5] M. G. Albrow et al., Nucl. Phys. B 108 (1976) 1; J. C. Armitage et al., Nucl. Phys. B 194 (1982)365.

[6] M. L. Good and W. D. Walker, Phys. Rev. 120 (1960) 1857.

[7] H. Miettinen and J. Pumplin, Phys. Rev. D 18 (1978) 1696. 